\title{
MODULARITY IN BIRKHOFF LATTICES
}

\section{R. WILCOX}

The purpose of this note is to identify upper semi-modular lattices originally defined by G. Birkhoff ${ }^{1}$ and subsequently studied by Dilworth ${ }^{2}$ with those $M$-symmetric lattices ${ }^{3}$ (introduced independently by the author without assumption of chain conditions) which satisfy a condition of finite dimensionality.

The definitions and notations are these. In a lattice $L, a>b(b<a)$ means that $a$ "covers" $b$, that is, $a>b$, together with $a \geqq x \geqq b$ implies $x=a$ or $x=b ;(b, c) M$ means $(a+b) c=a+b c$ for every $a \leqq c$ (where $a+b, a b$ are the "join" and "meet" respectively of $a, b)$. We say that $L$ is $M$-symmetric if the binary relation $M$ is symmetric; $L$ is a Birkhoff lattice if

$$
a, b>a b \text { implies } a+b>a, b \text {; }
$$

$L$ is of finite-dimensional type $e^{4}$ if for every $a<b$ there exists a finite "principal chain"

$$
a_{1}<a_{2}<\cdots<a_{n},
$$

with $a_{1}=a, a_{n}=b$. When $a, b$ satisfy this condition for a specific $n$, we say that $b$ is $n-1$ steps over $a$.

The properties of the relation $M$ are given in part in a previous paper. ${ }^{5}$ Additional properties needed here are contained in the following lemma.

Lemma 1. Suppose $b, c \in L$. Then

(a) $(b, c) M$ if and only if $b c \leqq a \leqq c$ implies $(a+b) c=a$;

(b) if $(b, c) M$, then $\left(b^{\prime}, c^{\prime}\right) M$ for $b c \leqq b^{\prime} \leqq b, b c \leqq c^{\prime} \leqq c$.

PROOF. The forward implication in (a) is obvious. To prove the

Presented to the Society, November 27, 1943; received by the editors November 22, 1943.

1 G. Birkhoff, Lattice theory, Amer. Math. Soc. Colloquium Publications vol. 25, New York, 1940, p. 62.

${ }^{2}$ R. P. Dilworth, Ideals in Birkhoff lattices, Trans. Amer. Math. Soc. vol. 49 pp. 325-353; also The arithmetical theory of Birkhoff lattices, Duke Math. J. vol. 8 (1941) pp. 286-299.

${ }^{8}$ L. R. Wilcox, Modularity in the theory of lattices, Ann. of Math. vol. 40 (1939) pp. 490-505; see also $A$ note on complementation in lattices, Bull. Amer. Math. Soc. vol. 48 (1942) pp. 453-457.

4 This property is weaker than finite dimensionality as used by Birkhoff (loc. cit. p. 11), even if 0 and 1 exist.

${ }^{5}$ L. R. Wilcox, Modularity in the theory of lattices, pp. 491-495. 
converse, let $a \leqq c$. Then $a^{\prime}=a+b c$ has the property $b c \leqq a^{\prime} \leqq c$, whence

$$
\begin{aligned}
(a+b) c & =(a+b c+b) c=\left(a^{\prime}+b\right) c \\
& =a^{\prime}=a+b c .
\end{aligned}
$$

To prove (b) we use the condition in (a). Let $b^{\prime} c^{\prime} \leqq a \leqq c^{\prime}$. Then

$$
\left(a+b^{\prime}\right) c^{\prime} \leqq(a+b) c c^{\prime}=a c^{\prime}=a \leqq\left(a+b^{\prime}\right) c^{\prime},
$$

whence (b) follows.

\section{THEOREM 1. Every $M$-symmetric lattice is a Birkhoff lattice.}

Proof. Suppose $a, b>a b$. Then it is immediate that $a+b>a, b$. To prove $a+b>a$, let $a \leqq c \leqq a+b$. Since $b \geqq c b \geqq a b$, we have $c b=b$ or $c b=a b$ from the hypothesis $b>a b$. If $c b=b$, then $a+b \leqq c$, whence $c=a+b$. Suppose $c b=a b$. We shall prove $(c, b) M$. Let $a b=c b \leqq x \leqq b$. Then $x=a b$ or $x=b$, whence either

or

$$
(x+c) b=(a b+c) b=(c b+c) b=c b=x,
$$

$$
(x+c) b=(b+c) b=b=x,
$$

and it follows by Lemma 1 (a) that $(c, b) M$. Now the symmetry of $M$ yields $(b, c) M$, and thus, since $b c \leqq a \leqq c$,

$$
c=(a+b) c=a \text {. }
$$

In all cases $c=a+b$ or $c=a$, and consequently $a+b>a$. Similarly $a+b>b$.

REMARK. The theorem just proved generalizes the known result ${ }^{6}$ that every modular lattice is a Birkhoff lattice, since modular lattices are $M$-symmetric.

In order to consider the converse of Theorem 1 , let, for the purposes of the following lemmas, $L$ be a fixed Birkhoff lattice of finitedimensional type.

LEMMA 2. If $b, c \in L$ and $c>b c$, then $b+c>b$.

Proof. ${ }^{7}$ Observe that $b \geqq b c$; if $b=b c, b \leqq c$, and $b+c=c>b c=b$. If $b>b c$, then there exists $n=1,2, \cdots$ such that $b$ is $n$ steps over $b c$. If $n=1$, the result is obvious from condition (1) defining a Birkhoff lattice. Suppose the result has been proved for all $b, c$ for which $b$ is

6 Birkhoff, loc. cit. p. 34 .

7 This is MacLane's second "exchange axiom" in the convex lattice of all $x \geqq b c$; as such it follows for finite-dimensional lattices from remarks on p. 63 of Birkhoff. Since $L$ need not be finite-dimensional, we give the proof in full. 
$k$ steps over $b c$, and let $b$ be $k+1$ steps over $b c$. Clearly there exists $b^{\prime}<b$ such that $b^{\prime}$ is $k$ steps over $b c$. Since $b^{\prime} c \leqq b c \leqq b^{\prime} c$, we have $c>b^{\prime} c$, and by the induction hypothesis applied to $b^{\prime}, c$ it follows that $b^{\prime}+c>b^{\prime}$. But $b^{\prime} \leqq\left(b^{\prime}+c\right) b \leqq b$, whence $\left(b^{\prime}+c\right) b=b^{\prime}$ or $\left(b^{\prime}+c\right) b=b$. In the latter case $b^{\prime}\left\langle b \leqq b^{\prime}+c\right.$, and thus $b=b^{\prime}+c$, whence $c \leqq b$, contrary to $c>b c$. Consequently $\left(b^{\prime}+c\right) b=b^{\prime}$. Since $b^{\prime}+c, b>\left(b^{\prime}+c\right) b$, (1) yields

$$
b+c=b+\left(b^{\prime}+c\right)>b .
$$

Lemma 3. If $b, c \in L, c>b c$, then $(c, b) M,(b, c) M$.

Proof. If $b c \leqq a \leqq c$, then $a=b c$ or $a=c$, so that either

or

$$
(a+b) c=(b c+b) c=b c=a,
$$

$$
(a+b) c=(c+b) c=c=a,
$$

and $(b, c) M$. Now suppose $b c \leqq a \leqq b$. Then $b c \leqq a c \leqq b c$ yields $a c=b c$. Hence $c>a c$, and $a+c>a$ by Lemma 2. But $a \leqq(a+c) b \leqq a+c$, whence $(a+c) b=a$ or $(a+c) b=a+c$. In the latter case $a+c \leqq b$, and $c \leqq b$, which is impossible. Hence $(a+c) b=a$, and $(c, b) M$.

Lemma 4. Suppose $b, c \in L,(b, c) M$. Then $b c \leqq a \leqq b, a+c=b+c$ implies $a=b$.

Proof. If $c=b c$, that is, $c \leqq b$, or if $c$ is one step over $b c$ then $(c, b) M$ either by direct verification or by Lemma 3 ; hence

$$
a=a+c b=(a+c) b=(b+c) b=b .
$$

Suppose the result holds for all $b, c$ with $c n$ steps over $b c$, and let $b, c$ satisfy the hypotheses, $c$ being $n+1$ steps over $b c$. Then there exists $c^{\prime}$ with $b c \leqq c^{\prime}\left\langle c\right.$, where $c^{\prime}$ is $n$ steps over $b c$. Since $\left(b, c^{\prime}\right) M$ by Lemma 1 (b), and since $b c^{\prime}=b c \leqq a \leqq b$, we need only verify $a+c^{\prime}$ $=b+c^{\prime}$ in order to show $a=b$. Since $(a, c) M$ by Lemma 1 (b), $\left(c^{\prime}+a\right) c=c^{\prime}$. Thus

$$
c>c^{\prime}=\left(c^{\prime}+a\right) c,
$$

and by Lemma 2 ,

$$
c+b=c+a=c+\left(c^{\prime}+a\right)>c^{\prime}+a .
$$

But

whence

$$
c^{\prime}+a \leqq c^{\prime}+b \leqq c+b,
$$

$$
c^{\prime}+b=c^{\prime}+a \text { or } c^{\prime}+b=c+b .
$$

In the second case, since $(b, c) M$, 


$$
c^{\prime}=\left(c^{\prime}+b\right) c=(c+b) c=c,
$$

which is impossible. This completes the proof.

THEOREM 2. Every Birkhoff lattice $L$ of finite-dimensional type is $M$-symmetric.

Proof. Suppose $(b, c) M$, and in proof of $(c, b) M$ let $b c \leqq a \leqq b$. Define

$$
b_{1}=(a+c) b \geqq a ;
$$

we shall prove that $b_{1}=a$ by applying Lemma 4 to $a, b_{1}, c$ in place of $a, b, c$. First, $\left(b_{1}, c\right) M$ by Lemma 1 (b), since $b c \leqq b_{1} \leqq b$, and $(b, c) M$. Moreover,

$$
b_{1} c=(a+c) c b=b c \leqq a \leqq b_{1} .
$$

Finally, $a+c \geqq b_{1}, c$, whence

$$
a+c \geqq b_{1}+c \geqq a+c,
$$

and $a+c=b_{1}+c$. The hypotheses of Lemma 4 have been verified, and thus $a=b_{1}$, as was to be proved.

The effect of Theorems 1 and 2 is to show that not necessarily finite-dimensional $M$-symmetric lattices are a true generalization of the Birkhoff lattices. Moreover, the condition defining $M$-symmetry does not lose its strength in infinite-dimensional cases as does condition (1). For example, an interval of real numbers ordered as usual satisfies (1) vacuously; it is modular, hence $M$-symmetric. However, define a lattice $L$ as consisting of the closed real interval $I=[0,1]$, ordered naturally, together with an element $\epsilon$, with $0<\epsilon<1$, but $x \nless \epsilon, \epsilon \nless x, \epsilon \neq x$ for $x \in I$. This is a lattice in which the only covering relations are $\epsilon>0,1>\epsilon$. Hence (1) is vacuously true, but $M$-symmetry fails violently, since $(x, \epsilon) M$ for every $x \in L$, but $(\epsilon, x) M$ is false except for $x=0,1$ or $\epsilon$.

Interesting questions are these. What infinite-dimensional generalization of the Jordan chain condition holds in $M$-symmetric lattices? Moreover, in finite-dimensional lattices, (1) together with its dual implies modularity; what can be said generally of lattices which together with their duals are $M$-symmetric?

ILLINOIS INSTITUTE OF TECHNOLOGX 